\title{
Multi-linear Regression Model to Predict the Electron Stability of Poorly Soluble Active Pharmaceutical Ingredients
}

\author{
Mark S'ari ${ }^{1}$, Andy Brown ${ }^{1}$, Rik Brydson ${ }^{1}$, Nicole Hondow ${ }^{1}$, Helen Blade ${ }^{2}$, Les Hughes ${ }^{2}$, Steve \\ Cosgrove $^{2}$ \\ 1. University of Leeds, School of Chemical and Process Engineering, Leeds, United Kingdom \\ 2. Pharmaceutical Development Group, AstraZeneca, Macclesfield, United Kingdom
}

Characterisation of active pharmaceutical ingredients (APIs) is important for drug processing and formulation, as many APIs crystallise into different structures, a phenomenon known as polymor-phism. Differences in crystal forms can lead to a changes in solid-state properties which depend on structure. Drug formulations may require a certain polymorph due to its properties, if a different alternative structure is present efficacy of the formulation may decrease or possible side effects can occur. Low percentage amounts of impurities $(<1 \%)$ are difficult to identify by conventional techniques such as powder X-ray Diffraction (pXRD) [1].

Transmission Electron Microscopy (TEM) can be used to identify the presence of different poly-morphs when other techniques fail [1,2]. The high spatial resolution of TEM allows specific areas and particles to be characterised through imaging and indexing of the electron diffraction patterns. However, these organic compounds are susceptible to damage induced by high energy electrons. This damage can be observed and measured from the fading of diffraction spots in the electron diffraction pattern, showing the eventual amorphisation of the crystal, as shown in Figure 1(a)(b). The aim of this study is to produce an empirical model based on twenty poorly soluble APIs that can predict the critical dose $\left(\mathrm{C}_{\mathrm{d}}\right)$ of other poorly soluble APIs from their molecular structures, thereby enabling screening of other poorly soluble compounds that may be suitable for TEM analysis.

All samples were provided by AstraZeneca and prepared for TEM by suspending the powders in water and placing droplets onto a continuous carbon TEM grid. The grids were examined by TEM (FEI Tecnai F20), operated at $200 \mathrm{kV}$. An electron flux of $0.003-0.061 \mathrm{e}^{-} / \mathrm{A}^{\circ} \mathrm{s}$ was used to measure the $\mathrm{C}_{\mathrm{d}}$ of the twenty compounds by measuring the intensity of first order diffraction spots over time [3]. The point at which the intensity drops to $1 / \mathrm{e}$ is considered the $C_{d}$, a decay graph of normalised intensity versus the electron fluence is shown in Figure 1(c).

The predictive model used multi-linear regression (MLR) analysis to correlate $C_{d}$ to a set of six predictor variables, chosen due to probable influence on $\mathrm{C}_{\mathrm{d}}$. Backwards regression, which removes predictor variables that have little effect on the model, was used to test which predictors are the most important. These were the ratio of aromatic carbons to aliphatic carbons $\left(\mathrm{C}_{\text {aromaliph }}\right)$ and ratio of hydrogen bond donors to acceptors $\left(\mathrm{HB}_{\mathrm{d}}: \mathrm{HB}_{\mathrm{a}}\right)$.

The results of the regression analysis gave an average $\mathrm{R}^{2}$ value of 0.52 and the equation:

$$
C_{d}=3.52+\left(1.36 \times C_{\text {arom:aliph }}\right)-\left(7.52 \times H B_{d}: H B_{a}\right)
$$

When using equation 1 to predict the $\mathrm{C}_{\mathrm{d}}$ and then comparing to the measured $\mathrm{C}_{\mathrm{d}}$ (Figure 2), compounds with higher measured $\mathrm{C}_{\mathrm{d}}$ appear harder to predict, as well as compounds that have no aromatic carbons. Previous studies have suggested that organic compounds containing conjugated 
ring systems are more stable to electron beam damage due to higher resonance stabilisation [4]. Similarly replacing hydrogen atoms with halides has been reported to increase stability [5]. Although the number of halogen atoms were removed as a predictor during this analysis, due to lack of significance.

The reason $\mathrm{HB}_{\mathrm{d}}: \mathrm{HB}_{\mathrm{a}}$ and $\mathrm{C}_{\text {aromaliph }}$ are the most significant predictors might be explained due to the intermolecular forces that occur within organic crystals, such as van der Waals forces, hydrogen bonding and $\pi-\pi$ stacking. All of these forces influence the lattice energy of organic crystals, hence impact on the amount of energy required to disrupt the crystal structure.

\section{References:}

[1] M D Eddleston et al, Journal of Pharmaceutical Sciences 99 (2010), p. 4072.

[2] R G Ricarte et al. Molecular Pharmaceutics 12 (2015), p. 983.

[3] M S'ari et al. Journal of Physics: Conference Series 644 (2015).

[4] W Jones Surface and Defect Properties of Solids 5 (1976), p. 65.

[5] W R K Clark et al. Ultramicroscopy 5 (1980), p. 195.
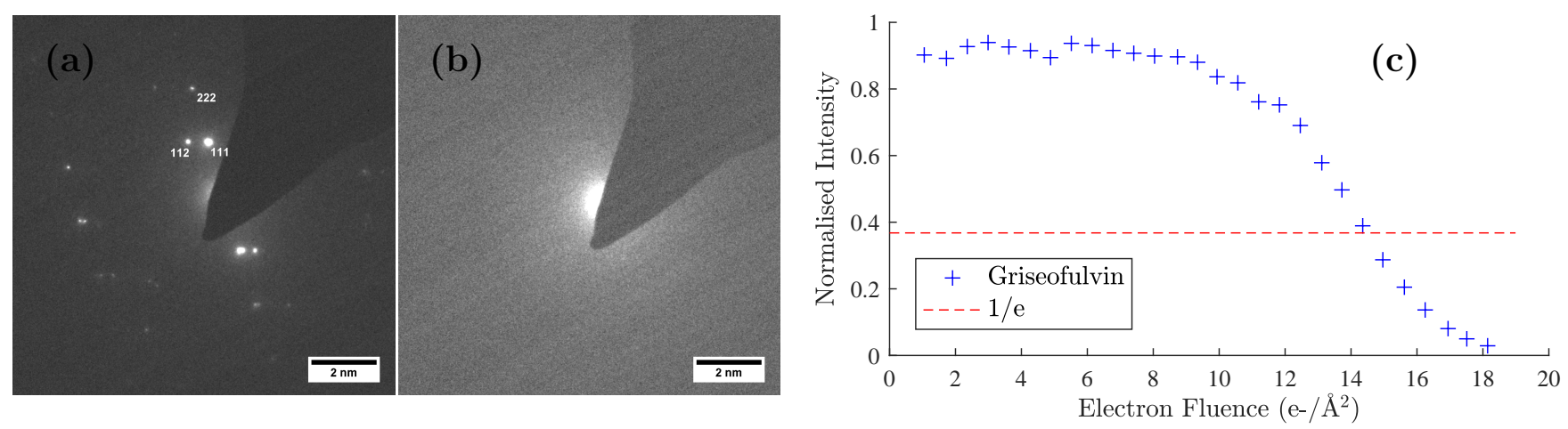

Figure 1. (a) Electron diffraction pattern $<110>$ zone axis of griseofulvin $50 \mathrm{~s}$ after the initial exposure (flux of 0.02 $\mathrm{e}^{-} / \mathrm{A}^{2} \mathrm{~s}$ ). (b) Diffraction pattern $920 \mathrm{~s}$ after initial exposure (total dose of $18.4 \mathrm{e}^{-} / \mathbb{A}^{\circ}$ ), showing amorphisation of griseofulvin. (c) Decay graph of the normalised intensity from griseofulvin (111) diffraction spot as a function of electron fluence, $C_{d}$ is where the decay reaches $1 /$ e of the initial intensity.

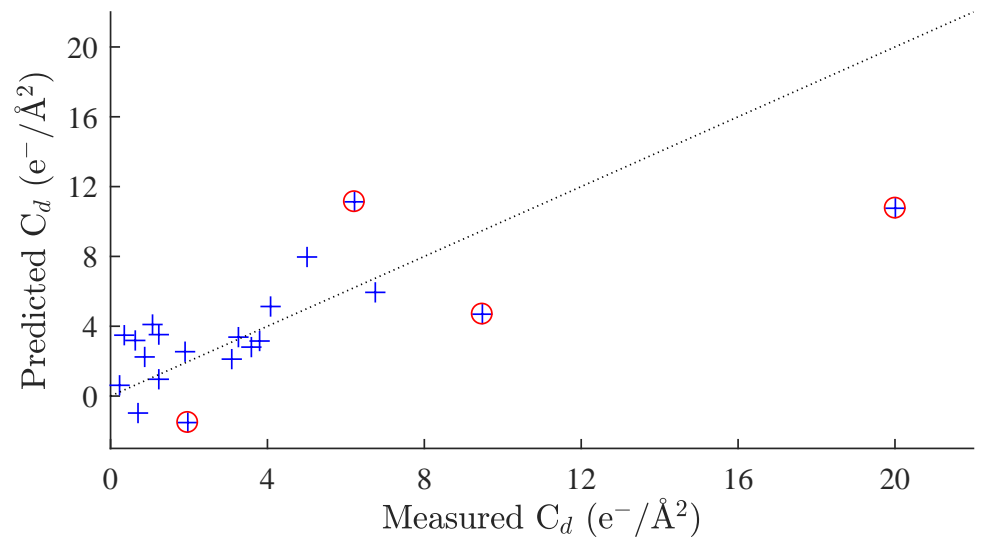

Figure 2. Predicted $C_{d}$ against measured $C_{d}$ values, calculated from graphs similar to the one in Figure 1c, the points highlighted in red circles show large errors in prediction, while the rest lie close to the regression model. 